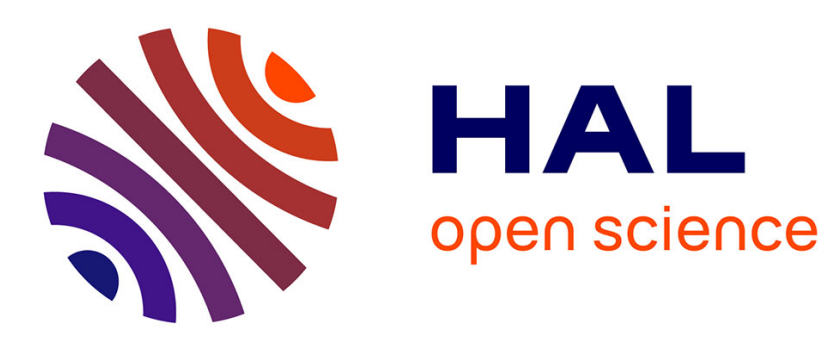

\title{
Quantitative approach to breather pair appearance in nonlinear modulational instability
}

Stefano Trillo, Matteo Conforti

\section{To cite this version:}

Stefano Trillo, Matteo Conforti. Quantitative approach to breather pair appearance in nonlinear modulational instability. Optics Letters, 2019, 44 (17), pp.4275. 10.1364/OL.44.004275 . hal-02384510

\author{
HAL Id: hal-02384510 \\ https://hal.science/hal-02384510
}

Submitted on 5 Dec 2019

HAL is a multi-disciplinary open access archive for the deposit and dissemination of scientific research documents, whether they are published or not. The documents may come from teaching and research institutions in France or abroad, or from public or private research centers.
L'archive ouverte pluridisciplinaire HAL, est destinée au dépôt et à la diffusion de documents scientifiques de niveau recherche, publiés ou non, émanant des établissements d'enseignement et de recherche français ou étrangers, des laboratoires publics ou privés. 


\title{
Quantitative approach to breather pair appearance in nonlinear modulational instability
}

\author{
Stefano Trillo ${ }^{1, *}$ and Matteo Conforti ${ }^{2}$ \\ ${ }^{1}$ Department of Engineering, University of Ferrara, Via Saragat 1, 44122 Ferrara, Italy \\ 2 Univ. Lille, CNRS, UMR 8523-PhLAM-Physique des Lasers Atomes et Molécules, F-59000 Lille, France \\ *Corresponding author: stefano.trillo@unife.it
}

Compiled August 1, 2019

\begin{abstract}
We investigate the generation of symmetric and asymmetric pairs of breathers from localized disturbances in the nonlinear stage of modulational instability governed by the nonlinear Schrödinger equation. An asymptotic matching approach allows us to predict with great accuracy how their emergence and their evolution depend on the parameters of the perturbation. The same approach turns out to be applicable to other classes of perturbations, which, due to their different decay in time, do not give rise to breathers.
\end{abstract}

http://dx.doi.org/10.1364/XX.XX.XXXXXX

Modulation instability (MI) involves the exponential growth of perturbations with sufficiently low frequency on top of a strong background (pump) [1-3]. Establishing how induced MI evolves past the initial amplification stage of the perturbation is the object of current intense efforts with main focus on experiments, which can be successfully described by the integrable nonlinear Schödinger equation (NLSE). For purely periodic perturbations, usually produced in experiments by seeding a pair of unstable sidebands, the nonlinear stage is characterized by longitudinal recurrences (cycles of conversion and back-conversion to the initial condition), which are a manifestation of the general scenario discovered by Fermi-Pasta-Ulam-Tsingou [4-8]. Such recurrences are, however, highly non-trivial, reflecting a breaking of symmetry and a consequent phase-space structure of the NLSE associated with the continuation of MI into the fully nonlinear regime $[9,10]$. Such features have been recently revealed in a fiber optics experiment $[6,7]$ as well as in a spatial experiment performed in regime of Kerr nonlinearity [8]. Importantly for what follows, a quantitative description of the recurrences can be obtained through asymptotic matching expansion as proposed by Grinevich and Santini [11], and fully justified by the application of rigorous finite-gap method under the only assumption of small input modulation [12]. Such result constitutes the proper generalization of a previous approach [13] that involves matching the exponentially growing modulation to the so-called Akhmediev breather (AB) [9].

Conversely, when the periodic perturbation is weighted by a bell-shaped envelope, thereby being localized, the multiple recurrence scenario breaks down. In this case, as discussed recently in [14], MI is responsible for the onset of an auto-modulation, i.e. a modulated structure similar to a dispersive shock wave [15], in a wedge-shaped region [16-19]. For perturbations decaying exponentially in time, this structure may coexist with a pair of breathers, i.e. solitons on background with internal breathing, also called Tajiri-Watanabe [20] or super-regular breather pairs [21-24]. In terms of the inverse scattering transform of the NLSE, auto-modulations and breathers are associated with continuous and discrete (point) spectrum of the direct scattering problem with non-zero boundary conditions, respectively [14, 16, 22].

In fiber optics, the breather pairs have been observed under ad hoc excitation (initial datum that fits the soliton pair interference pattern [23]), while the automodulation has been recently observed in the limit of zero central frequency of the perturbation [19]. Conversely the most general scenario of coexistence was not observed yet. In order to properly design the next generation of experiments it is of crucial importance to be able to predict with simple formulas how the distance of appearance of the breather pairs and their asymptotic velocities depend on the specific parameters of a sufficiently wide class of perturbations. In this Letter, we show that a sufficiently accurate answer in a wide range of parameters can be given by generalizing the asymptotic matched expansion of Ref. [11] to localized perturbations which decay slowly in time. Not only the method allows to predict with good accuracy the parameters of the emerging breathers, but gives also the correct description of the first appearance of coherent (rogue-wave like) structures for non-exponential perturbation which do not support the formation of breathers.

We consider the following NLSE conveniently written in dimensionless notation valid for fiber optics

$$
i \psi_{z}+\psi_{t t}+2|\psi|^{2} \psi=0,
$$

and investigate the evolution on top of a constant solution (pump) $\psi_{c w}=\psi_{0} \exp \left(i 2\left|\psi_{0}\right|^{2} z\right)$ of a localized modulation perturbation $\delta \psi(z, t)$ that initially possess the form

$$
\delta \psi(0, t)=\left[x_{1}(0, t) \exp (i \omega t)+x_{-1}(0, t) \exp (-i \omega t)\right],
$$

where $\omega$ is the modulation frequency, and $x_{ \pm 1}(0, t)=$ $a_{ \pm 1} \exp \left(-i \phi_{p}\right) f_{p}\left(t / t_{0}\right)$ describe weighting localized envelopes with duration $t_{0}$, peak amplitude $a_{ \pm 1}$, relative phase $\phi_{p}$, and shape $f_{p}\left(t / t_{0}\right)$, with $f_{p}\left(t / t_{0}\right) \geq 0, \max \left(f_{p}\right)=1$, and $f_{p}( \pm \infty)=$ 0 . Henceforth, without loss of generality, we take $\psi_{0}=1$, which amounts to express real-world propagating field, distance, and time in terms of real-world pump power $P=|E|^{2}$ 
as $E(Z, T)=\psi(z, t) \sqrt{P}, Z=2 z(\gamma P)^{-1}$ and $T=t \sqrt{\left|\beta_{2}\right| /(\gamma P)}$, $\beta_{2}<0$ and $\gamma$ being dispersion and effective nonlinear coefficient, respectively.

The physical idea behind the extension of the asymptotic matching to localized non-periodic perturbations is as follows. We assume the envelope to vary slowly compared to the modulation period (i.e., $t_{0} \gg 1 / \omega$ ) so that the input modulation grows locally (in time) due to MI according to its frequency $\omega$, phase $\phi_{p}$ and local instantaneous amplitude $a_{ \pm 1} f_{p}\left(t / t_{0}\right)$ at each time $t$. In order to determine at which distance for each $t$, say $z_{1}(t)$, such growth arrives at its apex, we match the linearized solution to a family of ABs having a slow dependence of the arbitrary shifts on local time $t$. Formally, the starting point is the following linearized equation obeyed by the perturbation $\delta \psi(z, t)$, obtained by retaining linear terms in Eq. (1) after substitution of the ansatz $\psi=\left[\psi_{0}+\delta \psi(z, t)\right] \exp \left(i 2\left|\psi_{0}\right|^{2} z\right)$

$$
i \delta \psi_{z}+\delta \psi_{t t}+2 \psi_{0}^{2} \delta \psi^{*}+2\left|\psi_{0}\right|^{2} \delta \psi=0
$$

We solve Eq. (3) with initial condition (2) by neglecting the time derivatives of the envelope $f_{p}\left(t / t_{0}\right)$, which reflects the assumption of slowly varying envelope, valid for $t_{0} \gg 1 / \omega$. Under such conjecture the solution can be easily calculated and depends on two eigenvalues $\lambda^{ \pm}= \pm g(\omega), g(\omega)=\omega \sqrt{4-\omega^{2}}$ being the MI amplitude gain, which are real in the MI bandwidth $|\omega| \leq 2$. Instead of dealing with $\omega$ as a parameter, it is convenient to express the solution in terms of the angle $\phi_{\omega}$ such that $\cos \phi_{\omega}=\frac{\omega}{2}$. The linear solution of the initial value problem [Eqs. (3-2)] then reads as

$$
\begin{aligned}
\psi(z, t) & =e^{i 2 z}\left[1+x_{1}(z, t) e^{i \omega t}+x_{-1}(z, t) e^{-i \omega t}\right], \\
x_{1}(z, t) & =+\frac{1}{i g} e^{i \phi_{\omega}}\left[\alpha_{+}^{*}(t) e^{i \phi_{\omega}} e^{g z}+\alpha_{-}(t) e^{-i \phi_{\omega}} e^{-g z}\right], \\
x_{-1}(z, t) & =-\frac{1}{i g} e^{-i \phi_{\omega}}\left[\alpha_{+}(t) e^{i \phi_{\omega}} e^{g z}+\alpha_{-}^{*}(t) e^{-i \phi_{\omega}} e^{-g z}\right]
\end{aligned}
$$

where $\alpha_{ \pm}(t)$ represent the local projection of the initial condition (2) over the growing and decaying eigenvectors corresponding to eigenvalues $\lambda^{ \pm}$, say $\mathbf{e}_{ \pm}=\left[\exp \left( \pm i 2 \phi_{\omega}\right), 1\right]^{T}$, with $\exp \left( \pm i 2 \phi_{\omega}\right)=\frac{\omega^{2}-2 \pm i g(\omega)}{2}$. Explicitly $\alpha_{ \pm}(t)$ read as follows

$$
\alpha_{ \pm}(t)=x_{ \pm 1}^{*}(0, t)-e^{ \pm i 2 \phi_{\omega}} x_{\mp 1}(0, t) .
$$

Note that Eqs. (4-7) represents essentially the three-wave linearized solution obtained in the context of standard MI analysis (i.e., in the limit $t_{0} \rightarrow \infty$ ), except for an additional parametric dependence on time appearing through the terms $\alpha_{ \pm}(t)$ which contain the information on the input envelope shape.

The subsequent stage which involves saturated growth to the apex and following decay can be described by matching to the following AB solution of Eq. (1)

$$
\psi_{A B}(z, t)=\frac{\sin \theta \cos \left[\omega\left(t-t_{1}\right)\right]+\cosh \left[g\left(z-z_{1}\right)+i 2 \theta\right]}{\cosh \left[g\left(z-z_{1}\right)\right]-\sin \theta \cos \left[\omega\left(t-t_{1}\right)\right]} e^{i 2 z+i \rho},
$$

characterized by the parameter $\theta$, with $0 \leq \theta \leq \pi / 2$ ( $g=$ $2 \sin 2 \theta, \omega=2 \cos \theta$ ), and by the arbitrary shifts $t_{1}, z_{1}, \rho$. The $\mathrm{AB}$ is well known to describe a heteroclinic orbit connecting the background with different phase $\pm 2 \theta$ at $z= \pm \infty$ passing through apex of growth at $z=z_{1}$. Matching with the linearized solution, requires to consider the asymptotic limit of Eq. (8) for $z-z_{1} \rightarrow-\infty$, which yields

$$
\psi_{A B} \simeq e^{i 2 z}\left\{1+g e^{g\left(z-z_{1}\right)} e^{i \theta} \cos \left[\omega\left(t-t_{1}\right)\right]\right\} e^{-i 2 \theta+i \rho} .
$$

Due to the form of Eq. (9), it is convenient to cast (with straightforward algebra) the growing part of the solution in Eq. (4-5-6) in the following form

$$
\psi \simeq e^{i 2 z}\left\{1+\frac{2}{g}\left|\alpha_{+}(t)\right| e^{g z} e^{i \phi_{\omega}}\left[\omega\left(t-T_{\text {shift }}(t)\right)\right]\right\},
$$

where $T_{\text {shift }}(t) \equiv \frac{\arg \left[\alpha_{+}(t)\right]-\phi_{\omega}+\pi / 2}{\omega}$. Finally, comparing Eq. (10) with Eq. (9), we obtain that a family of $A B$ is needed to describe the growth process, which has fixed parameters $\theta=\phi_{\omega}$ and $\rho=2 \theta$, and slowly varying in time shifts $t_{1}$ and $z_{1}$ which are contrained to satisfy $t_{1}=T_{\text {shift }}(t)$ and $g \exp \left(-g z_{1}\right)=2\left|\alpha_{+}(t)\right| / g$. In particular, the latter relation gives

$$
z_{1}(t)=\frac{1}{g} \log \left[\frac{g^{2}}{2\left|\alpha_{+}(t)\right|}\right],
$$

which is main result of this paper, representing an analytical prediction of the general locus of apex growth in the plane $t-z$. For symmetric sidebands, i.e. a perturbation of the form $\delta \psi(0, t)=$ $a_{p} \exp \left(-i \phi_{p}\right) f_{p}\left(t / t_{0}\right) \cos (\omega t)$ with peak amplitude $a_{p}=2 a_{1}=$ $2 a_{-1}$, we obtain $\alpha_{+}(t)=\exp \left(i \phi_{\omega}\right) i a_{p} f_{p}\left(t / t_{0}\right) \sin \left(\phi_{p}-\phi_{\omega}\right)$, and hence a constant $t_{1}$, either $t_{1}=0$ for $-\pi<\phi_{p}-\phi_{\omega}<0$ or $t_{1}=\pi / \omega$ for $0<\phi_{p}-\phi_{\omega}<\pi$ (more generally, $t_{1}$ may vary locally with time if the input phase $\phi_{p}$ becomes time depedent), whereas Eq. (11) yields

$$
z_{1}(t)=\frac{1}{g} \log \left[\frac{g^{2}}{2 a_{p} f_{p}\left(t / t_{0}\right)\left|\sin \left(\phi_{p}-\phi_{\omega}\right)\right|}\right] .
$$

Since breathers traveling at constant velocity requires a linear asymptotic dependence of $z_{1}$ on $t$, Eqs. (11-12) quantitatively justify the fact that breathers require perturbation envelopes with exponential decay, e.g. $f_{p}=\operatorname{sech}\left(t / t_{0}\right)$ or $\exp \left(-|t| / t_{0}\right)$, as conjectured in [14]. Remarkably Eq. (12) describes the full dynamical stage from the first local apex appearance occurring at minimal distance $z_{\min }=\min \left[z_{1}(t)\right]$ to their asymptotic separation with velocities $\pm V_{s}$, which are determined by the decay law $\exp \left( \pm t / t_{0}\right)$ of envelope tails. From Eq. (12), we easily find

$$
V_{s}=g(\omega) t_{0} ; \quad z_{\min }=\frac{1}{g} \log \left[\frac{g^{2}}{2 a_{p}\left|\sin \left(\phi_{p}-\phi_{\omega}\right)\right|}\right] .
$$

In order to check the validity of Eqs. (11-12-13), we compare with numerical simulations of the NLSE. Figure 1 reports a typical example of false color plot of the NLSE evolution obtained for symmetric sidebands and sech-shaped envelope $\left(f_{p}=\operatorname{sech}\left(t / t_{0}\right)\right.$, parameters as reported in the caption). One clearly sees that a symmetric pair of breather is emitted, which coexists with an automodulation building up at larger distances and developing inside the wedge-shaped region delimited by the maximal group velocities $\pm V_{w}= \pm 4 \sqrt{2} \psi_{0}[14,16,18]$. Superimposed to the false color plot we report the locus of peak amplification of the breathers predicted by Eq. (12), see dashed white curve. As shown, the asymptotic matching perfectly fit with the local maxima of the breathers, in particular giving the correct minimal distance $z_{\text {min }}$ and asymptotic velocities $\pm V_{s}$, both well approximated by Eqs. (13). Here $z_{\min }$ is defined as the distance the modulation pattern undergoes the first peak (observed at $t=t_{1}=0$ in Fig. 1). Such agreement is obtained in a wide range of the parameters of the input modulation, with appreciable deviations appearing only for narrow envelopes such that $t_{0} \sim 2 \pi / \omega$ (e.g. $t_{0} \sim 3$ for $\omega=\sqrt{2}$ ). In Fig. 2(a), we summarize the dependence of the minimum distance $z_{\min }$ on 


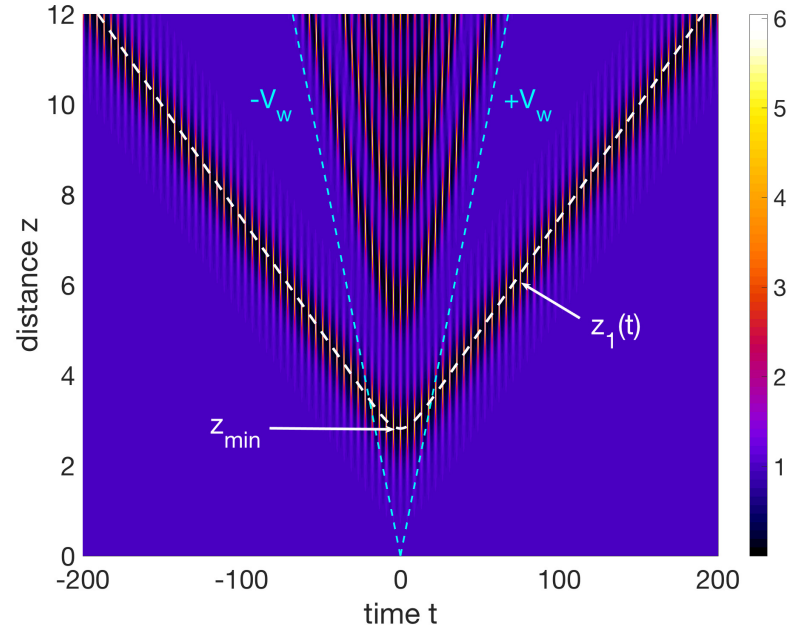

Fig. 1. Numerical solution of NLSE (1) with input (2), $\psi_{0}=1$, sech-shaped envelope with parameters: $a_{p}=0.01, \phi_{p}=-\pi / 2$, $t_{0}=10, \omega=\omega_{\text {peak }}=\sqrt{2}$. The dashed cyan lines stand for velocities $V= \pm V_{w}$ delimiting the automodulation wedge. The dashed white line stands for $z_{1}(t)$ from Eq. (12).
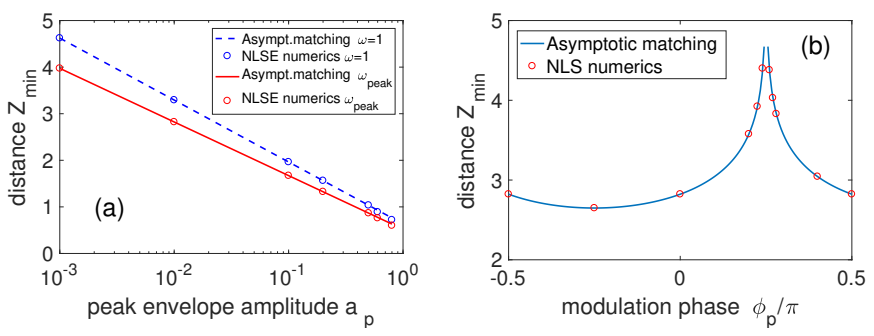

Fig. 2. Formation distance $z_{\min }$ (a) vs. peak modulation amplitude $a_{p}$, at fixed phase $\phi_{p}=-\pi / 2$, for frequencies $\omega=1$, $\omega_{\text {peak }}=\sqrt{2} ;(\mathrm{b})$ vs. phase $\phi_{p}$ with fixed amplitude $a_{p}=0.01$, $\omega=\sqrt{2}$. Here the envelope is sech-shaped with $t_{0}=10$. The asymptotic formula is compared with data from NLSE simulations (open dots).

the perturbation amplitude $a_{p}$ for two different modulation frequencies $\left(\omega=1, \omega_{\text {peak }}=\sqrt{2}\right)$, and fixed phase $\phi_{p}=-\pi / 2$. The two lines with different slopes, which correspond to $z_{\text {min }}$ in Eq. (13), are found in excellent agreement with values extracted from numerical solution of the NLSE (open dots) over a range that spans nearly three orders of magnitude $\left(a_{p}=10^{-3}-0.8\right)$. Noteworthy, the agreement remains reasonably good (error being less than $6 \%$ ) even when $a_{p}=0.8$, i.e. sidebands with amplitude comparable to the pump, where the theory is expected to loose accuracy. Moreover we have verified that the velocities $\pm V_{s}$ are unaffected by the change of amplitude over such a large span, as predicted by Eq. (13). Importantly, also the perturbation phase of the modulation is found to have a strong impact on the breather pair formation. This is shown in Fig. 2(b) where we compare $z_{\min }$ vs. perturbation phase $\phi_{p}$ from Eq. (12) to the NLSE numerics (open dots). The agreement is clearly excellent. In particular, there are two notable value of perturbation phase $\phi_{p}= \pm \phi_{\omega}\left[\phi_{\omega}=\pi / 4\right.$ for $\omega=\omega_{\text {peak }}$ as in Fig. 2(b)], which correspond, in the framework of the three-wave description of MI for periodic perturbation $[6,10]$, to evolution along the unsta- ble $\left(\phi_{p}=-\phi_{\omega}\right)$ or stable $\left(\phi_{p}=\phi_{\omega}\right)$ manifold of the separatrix, which represent the continuation into the fully nonlinear stage of the linear instability. The unstable manifold is characterized by the fastest growth to the apex and hence yields the absolute shortest distance $z_{m i n}$, as obtained in Fig. 2(b) consistently with Eq. (12). Conversely, for $\phi_{p}=\phi_{\omega}, z_{\min } \rightarrow \infty$ [see Eq. (12)] since the stable manifold is characterized by the modulation converting asymptotically its energy to the pump, thus requiring a virtually infinite distance to grow again. The NLSE simulations show that the distance $z_{\text {min }}$ increases by following the predicted trend as $\phi_{p}$ approaches $\phi_{\omega}$. However, the numerics indicates that it never really diverges, rather saturating around the typical distance where the auto-modulation appears [e.g. around $z_{\text {min }} \sim 4.5$ for the amplitude considered in Fig. 2(b)] thus acting as a seed for breather formation.

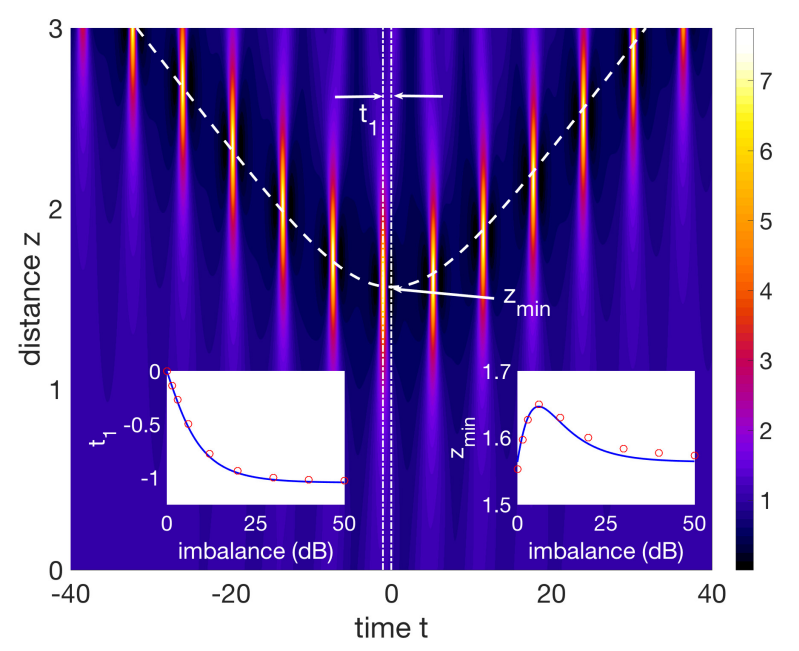

Fig. 3. As in Fig. 1, zoom on breathers emergence for asymmetric sidebands giving rise to a temporal shift $t_{1}$ of the first peak. Here $\omega=1, \phi_{p}=-\pi / 2, a_{1}=0.1, a_{-1}=0.001$, sechshaped envelope with $t_{0}=10$. The insets show how the shift $t_{1}$ and minimal distance $z_{\text {min }}$ depend on the sideband imbalance $20 \log _{10} a_{1} / a_{-1}$ (decibel units), with fixed $a_{1}=0.1$. The theoretical prediction (solid line) is compared with NLSE simulations (open dots).

Let us consider next the case of asymmetric sideband amplitudes $a_{1} \neq a_{-1}$. In the periodic case, this introduces a net drift velocity of the $A B$ [25]. However, for localized perturbation, as discussed before, the velocities $\pm V_{s}$ are unaffected by variations of sideband amplitudes. Hence, the theory still predicts emergence of breathers (provided that $f_{p}$ decays exponentially) with opposite asymptotic velocities $\pm V_{s}$, as observed numerically [see Fig. 3]. However, in this case, the broken sideband symmetry reflects itself in a shift $t_{1}$ which is no longer zero or $\pi / \omega$, but rather varies continuously with the imbalance between the sidebands. The typical scenario is illustrated in Fig. 3. The first peak emerges with a shift $t_{1}$ from the peak of the initial perturbation (at $t=0)$. The smooth variation of $t_{1}$ over large variation of sideband imbalance is well described by the theory as shown in the left inset in Fig. 3. The sideband imbalance has also a slight impact on the formation distance $z_{\min }$ (see right inset in Fig. 3), which, again, turns out to be well predicted by Eq. (11).

In order to have asymmetric breathers traveling with different velocities one needs to break the parity of the modulation 
envelope in Eq. (2). Maintaining exponential decay, this is obtained, for instance, for $f_{p}(t)=\exp \left(-|t| / t_{0 \pm}\right)$, with $t_{0+}, t_{0-}$ $\left(t_{0+} \neq t_{0-}\right)$ being durations for $t>0$ and $t<0$, respectively. An example is shown in Fig. 4 , where $t_{0+}=10$ and $t_{0-}=5$ are such that both breathers travel with different velocities outside the wedge. By choosing $t_{0 \pm}$ such that $\left|V_{s}\right|<\left|V_{w}\right|$, one can also observe interaction between breathers and auto-modulation as demonstrated in [14] (not shown here).

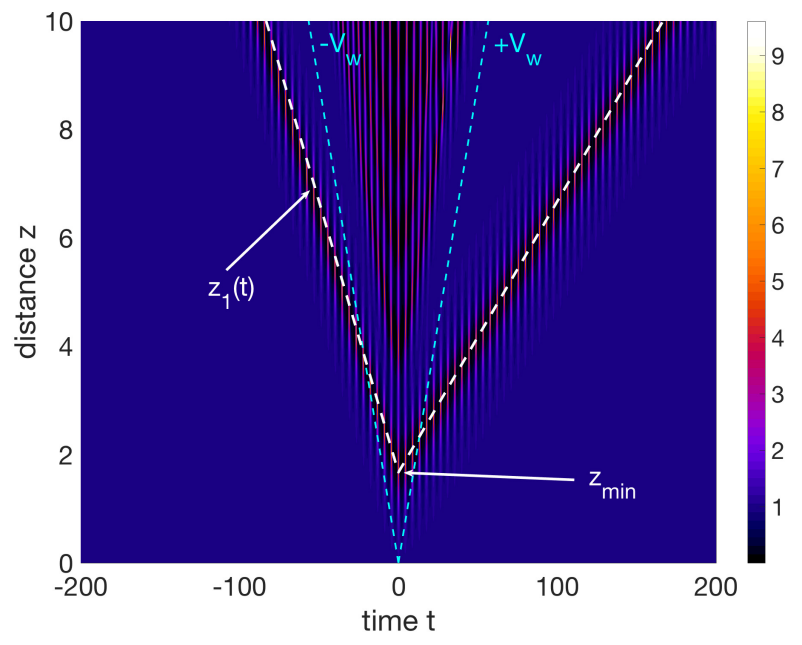

Fig. 4. Asymmetric breather pair generated with non-even envelope $f_{p}(t)=\exp \left(-|t| / t_{0 \pm}\right)$. Here $t_{0+}=10, t_{0-}=5$, $\omega=1, a_{p}=0.1, \phi_{p}=-\pi / 2$. Cyan dashed lines stand for velocities $\pm V_{w}$.

So far we have examined cases where the exponential decay of the perturbation supports breather pair formation. However, we emphasize that Eq. (11) has more general validity, describing the locus of the apex growth due to localized MI also for envelopes with decay laws such that no breathers are formed. We have tested such an idea for different envelope shapes, e.g. Gaussian pulses (as reported in [14]) or power-law decay $f_{p}\left(t / t_{0}\right)=1 /\left[1+\left(t / t_{0}\right)^{n}\right]$, always finding good agreement. As an example, we display in Fig. 5 the evolution relative to a Lorentzian pulse envelope, i.e. $n=2$ in the power-law decay. As shown, the dashed white line, which stands for the locus in $(t, z)$ plane corresponding to Eq. (12), suitably describes the peak conversion locus obtained from the numerics. Here the pulses have no soliton features and slightly broaden while exhibiting strong bending upon propagation. Despite its evident non-soliton character, Eq. (12) is still accurate, and in particular gives a quantitative description of the early formation point, which is still described by $z_{\min }(t)$ in Eq. (13). As a consequence, also in this case $z_{\min }(t)$ follows the dependence on perturbation amplitude and phase already discussed for the breather case, and summarized in Fig. 2.

In summary, by extending the asymptotic matching approach [11] to localized perturbations, we have shown that the emergence of breather pairs can be predicted with high accuracy by means of simple closed formulas. Such formula allows also for predicting the locus of pulse formation for perturbations which do not support breathers.

\section{FUNDING}

S.T. acknowledges discussions with P. Grinevich and P. Santini.

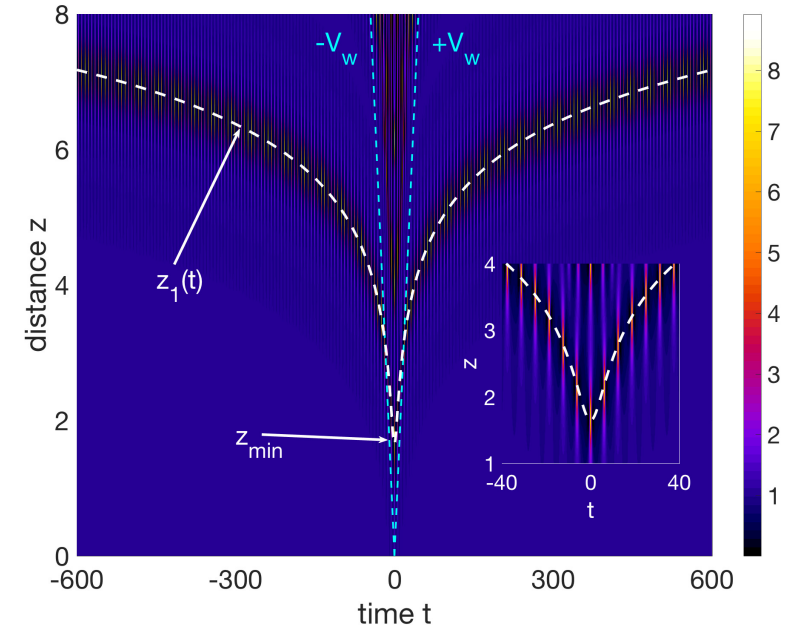

Fig. 5. Evolution of a localized perturbation with Lorentzian envelope. Here the parameters are $\omega=1, t_{0}=5, \phi_{1}=-\pi / 2$, $a_{p}=0.1$. Dashed white line stand for $z_{1}(t)$ from Eq. (11). Cyan dashed lines stand for velocities $\pm V_{w}$. The inset shows a zoom around $z_{\min }(t)$.

\section{REFERENCES}

1. V. I. Bespalov and V. I. Talanov, JETP Lett. 3, 307 (1966).

2. K. Tai, A. Hasegawa, and A. Tomita, Phys. Rev. Lett. 56, 135 (1986).

3. J. M. Dudley, F. Dias, M. Erkintalo, and G. Genty, Nature Photon. 8, 755 (2014).

4. G. Van Simaeys, Ph. Emplit, and M. Haelterman, Phys. Rev. Lett. 87, 033902 (2001).

5. A. Bendhamane, A. Mussot, P. Szriftgiser, A. Kudlinski, M. Conforti, S. Wabnitz, and S. Trillo, Opt. Exp. 23, 30861 (2015).

6. A. Mussot, C. Naveau, M. Conforti, A. Kudlinski, F. Copie, P. Szriftgiser, and S. Trillo, Nature Photon. 10, 303 (2018).

7. C. Naveau, P. Szriftgiser, A. Kudlinski, M. Conforti, S. Trillo, and A. Mussot, Opt. Lett. 44, 763 (2019).

8. D. Pierangeli, M. Flammini, L. Zhang, G. Marcucci, A. J. Agranat, P. G. Grinevich, P. M. Santini, C. Conti, and E. DelRe, Phys. Rev. X 8, 041017 (2018).

9. N. N. Akhmediev, and V. I. Korneev, Theor. Math. Phys. 69, 1089 (1987).

10. S. Trillo and S. Wabnitz, Opt. Lett. 16, 986 (1991).

11. P. G. Grinevich and P. M. Santini, Phys. Lett. A 382, 973 (2018).

12. P. G. Grinevich and P. M. Santini, Nonlinearity 31, 5258 (2018).

13. M. Erkintalo, G. Genty, B. Wetzel, and J. M. Dudley, Phys. Lett. A 375, 2029 (2011).

14. M. Conforti, S. Li, G. Biondini, and S. Trillo, Opt. Lett. 43, 5291 (2018).

15. G. El, A. Gurevich, V. Khodorovskii, and A. Krylov, Phys. Lett. A 177, 357 (1993).

16. G. Biondini and D. Mantzavinos, Phys. Rev. Lett. 116, 043902 (2016).

17. G. Biondini, S. Li and D. Mantzatinos, Phys. Rev. E 94, 060201R (2016).

18. G. Biondini, S. Li, D. Mantzavinos, and S. Trillo, SIAM Review 60, 888 (2018).

19. A. E. Kraych, P. Suret, G. El, and S. Randoux, Phys. Rev. Lett. 122, 054101 (2018).

20. M. Tajiri and Y. Watanabe, Phys. Rev. E 57, 3510 (1998).

21. V. E. Zakharov and A. Gelash, Phys. Rev. Lett. 111, 054101 (2013).

22. A. A. Gelash and V. E. Zakharov, Nonlinearity 27, 1 (2014).

23. B. Kibler, A. Chabchoub, A. A. Gelash, N. Akhmediev and V. E. Zakharov, Phys. Rev. X 5, 041026 (2015).

24. A. Gelash, Phys. Rev. E 97, 022208 (2018).

25. B. Frisquet, B. Kibler and G. Millot, Phys. Rev. X 3, 041032 (2013). 


\section{REFERENCES}

1. V. I. Bespalov and V. I. Talanov, Filamentary Structure of Light Beams in Nonlinear Liquids, JETP Lett. 3, 307 (1966).

2. K. Tai, A. Hasegawa, and A. Tomita, Observation of modulation instability in optical fibers, Phys. Rev. Lett. 56, 135 (1986).

3. J. M. Dudley, F. Dias, M. Erkintalo, and G. Genty, Instabilities, breathers and rogue waves in optics, Nature Photon. 8, 755 (2014).

4. G. Van Simaeys, Ph. Emplit, and M. Haelterman, Experimental demonstration of the Fermi-Pasta-Ulam recurrence in a modulationally unstable optical wave, Phys. Rev. Lett. 87, 033902 (2001).

5. A. Bendhamane, A. Mussot, P. Szriftgiser, A. Kudlinski, M. Conforti, S. Wabnitz, and S. Trillo, Optimal frequency conversion in the nonlinear stage of modulation instability, Opt. Exp. 23, 30861 (2015).

6. A. Mussot, C. Naveau, M. Conforti, A. Kudlinski, F. Copie, P. Szriftgiser, and S. Trillo, Fibre multiwave-mixing combs reveal the broken symmetry of Fermi-Pasta-Ulam recurrence, Nature Photon. 10, 303 (2018).

7. C. Naveau, P. Szriftgiser, A. Kudlinski, M. Conforti, S. Trillo, and A. Mussot, Full-field characterization of breather dynamics over the whole length of an optical fiber, Opt. Lett. 44, 763 (2019).

8. D. Pierangeli, M. Flammini, L. Zhang, G. Marcucci, A. J. Agranat, P. G. Grinevich, P. M. Santini, C. Conti, and E. DelRe, Observation of Fermi-Pasta-Ulam-Tsingou Recurrence and Its Exact Dynamics, Phys. Rev. X 8, 041017 (2018).

9. N. N. Akhmediev, and V. I. Korneev, Modulation instability and periodic solutions of the nonlinear Schrödinger equation, Theor. Math. Phys. 69, 1089 (1987).

10. S. Trillo and S. Wabnitz, Dynamics of the nonlinear modulational instability in optical fibers, Opt. Lett. 16, 986 (1991).

11. P. G. Grinevich and P. M. Santini, The exact rogue wave recurrence in the NLS periodic setting via matched asymptotic expansions, for 1 and 2 unstable modes, Phys. Lett. A 382, 973 (2018).

12. P. G. Grinevich and P. M. Santini, The finite gap method and the analytic description of the exact rogue wave recurrence in the periodic NLS Cauchy problem, Nonlinearity 31, 5258 (2018).

13. M. Erkintalo, G. Genty, B. Wetzel, and J. M. Dudley, Akhmediev breather evolution in optical fiber for realistic initial conditions, Phys. Lett. A 375, 2029 (2011).

14. M. Conforti, S. Li, G. Biondini, and S. Trillo, Auto-modulation versus breathers in the nonlinear stage of modulational instability, Opt. Lett. 43, 5291 (2018).

15. G. El, A. Gurevich, V. Khodorovskii, and A. Krylov, Modulational instability and formation of a nonlinear oscillatory structure in a focusing medium, Phys. Lett. A 177, 357 (1993).

16. G. Biondini and D. Mantzavinos, Universal nature of the nonlinear stage of modulational instability, Phys. Rev. Lett. 116, 043902 (2016).

17. G. Biondini, S. Li and D. Mantzatinos, Oscillation structure of localized perturbations in modulationally unstable media, Phys. Rev. E 94, 060201R (2016).

18. G. Biondini, S. Li, D. Mantzavinos, and S. Trillo, Universal behavior of modulationally unstable media, SIAM Review 60, 888 (2018).

19. A. E. Kraych, P. Suret, G. El, and S. Randoux, Phys. Rev. Lett. 122, 054101 (2018).

20. M. Tajiri and $Y$. Watanabe, Breather solutions to the focusing nonlinear Schrödinger equation, Phys. Rev. E 57, 3510 (1998).

21. V. E. Zakharov and A. Gelash, Nonlinear stage of modulation instability, Phys. Rev. Lett. 111, 054101 (2013).

22. A. A. Gelash and V. E. Zakharov, Superregular solitonic solutions: a novel scenario for the nonlinear stage of modulation instability, Nonlinearity 27, 1 (2014).

23. B. Kibler, A. Chabchoub, A. A. Gelash, N. Akhmediev and V. E. Zakharov, Superregular breathers in optics and hydrodynamics: Omnipresent modulation instability beyond simple periodicity, Phys. Rev. X 5, 041026 (2015).

24. A. Gelash, Formation of rogue waves from the locally perturbed condensate, Phys. Rev. E 97, 022208 (2018).

25. B. Frisquet, B. Kibler and G. Millot, Collision of Akhmediev breathers in 